\title{
Génétique du cancer colo-rectal
}

\section{Cancer du côlon et réparation de l'ADN}

médecine/sciences, dans son numéro d'août-septembre 1993, rapportait la découverte d'un gène de susceptibilité au cancer colique associé à une instabilité de l'ADN, gène localisé sur le bras court du chromosome 2 (en $2 p$ 15.16). Ce gène semblait responsable des formes familiales du cancer du colon non associées à une polypose colique (syndrome de Lynch, $m / s, n^{\circ} 8-9$, vol. 9, p. 990). Une observation extrêmement surprenante avait été faite au niveau de l'ADN des tumeurs survenant chez les malades atteints de cette forme de cancer colique: nombre de micro-satellites* sont de taille modifiée par rapport au tissu normal. Cette observation suggérait que le gène de susceptibilité pouvait intervenir dans la stabilité de l'ADN. C'est ce qui vient maintenant d'être démontré par l'équipe de K. Kinzler et B. Vogelstein (Baltimore, MA, USA) [1] ainsi que par celles de R. Fishel (Dorlington, VE, USA) et R. Kolodner (Harvard, MA, USA) [2]. Fishel et Kolodner sont partis de l'identification de l'équivalent humain d'un gène de réparation chez la levure, impliqué dans l'excision des nucléotides mal appariés, gène initialement identifié par l'équipe de Kolodner (gène Mut $S$ chez E.coli: et MSH2 chez la levure). Le gène humain ainsi identifié, $h M S H 2$, se révéla être localisé sur le bras court du chromosome 2, juste au locus auquel avait préalablement été assigné le gène de susceptibilité au cancer colique héréditaire sans polypose [2]. Quant à l'équipe de Kinzler et Vogelstein, associée à A. de la Chapelle (Helsinki, Fin-

\footnotetext{
* Répétition de dinucléotides (A, entourés de séquences uniques; le nombre de répétitions est très polymorphe, la taille des micro-satellites constituan par conséquent un marqueur génétique extrêmement
}

lande) et à J. Trent (Bethesda, MD, USA), ils arrivèrent au gène par la méthode de clonage positionnel. Dans l'intervalle auquel ils parvinrent, se trouvait l'équivalent humain du gène Mut $S[1]$. Les deux équipes démontrèrent alors qu'existaient des mutations constitutionnelles d'un allèle du gène Mut $S$ chez les malades ayant cette susceptibilité au cancer colique. Dans les tumeurs, cette mutation hétérozygote du gène $M u t$ $S$ devient homo ou hémizygote par perte d'hétérozygotie [1], rendant les cellules tumorales cent fois plus sensibles à des mutagènes que des cellules normales [3]. La responsabilité du gène $h M S H 2$ dans l'instabilité des répétitions CA des microsatellites est rendue très probable par le phénotype des levures déficientes, msha -/-, qui comporte une semblable instabilité de répétitions de dinucléotides [2]. En outre, l'introduction dans $E$. coli du gène $h M S H 2$ produit un phénotype hypermutateur, probablement du fait de l'interaction aberrante entre le produit de ce gène et les protéines de E. coli participant à la voie de réparation de l'ADN impliquant Mut S[2].

La découverte de ces deux gènes de susceptibilité est évidemment extrêmement importante, tant sur le plan fondamental (il confirme l'un des mécanismes importants de susceptibilité au cancer, c'est-à-dire l'anomalie des systèmes de réparation de l'ADN) que pratique: cette forme de cancer est très fréquente (jusqu'à $15 \%$ de tous les cancers coliques) et le gène anormal pourrait être porté par une personne sur 200, posant le problème d'un criblage de masse possible. Dans ce cas, la détection d'une prédisposition semble particulièrement intéressante puisque la détection précoce d'un cancer débutant, telle qu'elle pourrait être effectuée chez les sujets à risques, permet pratiquement d'assurer la guérison des malades après exérèse chirurgicale de la tumeur.
Le produit du gène $A P C$ de la polypose colique familiale est associé à des protéines d'ancrage du cytosquelette

Le gène $A P C$, localisé sur le chromosome 5, est modifié dans la polypose colique familiale et dans de nombreux autres types de cancers digestifs dans lesquels cette mutation semble être un phénomène très précoce $\left(m / s, n^{\circ} 7\right.$, vol. 7, p. $778 ; n^{\circ} 6$, vol. 8, p. 607; $n^{\circ}$ 9, vol. 8, p. 1000). Deux équipes américaines, l'une de Baltimore (MD) [4] et l'autre de Richmond, Emeryville et San Francisco (CA) [5] viennent maintenant de démontrer une interaction forte entre un motif de 15 acides aminés de la protéine APC et les caténines $\beta[4,5]$ et $\alpha$ [4]. Les caténines sont des protéines localisées au niveau des jonctions adherens des épithéliums, structures assurant la stabilité de ces tissus et jouant également, probablement, un rôle dans l'inhibition de contact. Ces jonctions adherens sont constituées de protéines d'adhérence transmembranaires, les cadhérines, dont la portion extracellulaire comporte des motifs de type immunoglobulines [6] qui interviennent dans des interactions homotypiques entre les cadhérines de cellules contiguës. Par leur domaine intracellulaire, les cadhérines interagissent avec les caténines $\alpha$ et $\beta$ auxquelles les faisceaux d'actine du cytosquelette semblent ancrés. L'intervention des caténines dans la transmission d'un signal de l'extérieur vers l'intérieur de la cellule, peut-être impliqué dans l'inhibition de contact, est également suggérée par les conséquences des mutations du gène armadillo chez la drosophile. Le produit de ce gène, un homologue de la caténine $\beta$, est en effet indispensable à la transmision du signal passant par le produit du gène wingless et intervenant dans la formation de la cuticule embryonnaire de drosophile [7]. Les relations entre le 
cytosquelette et les molécules d'adhérence intercellulaires, d'une part, et le cancer, d'autre part, sont maintenant bien établies. Des modifications des molécules d'adhérence peuvent faciliter le développement de métastases $\left(\mathrm{m} / \mathrm{s}, n^{\circ} 6 / 7\right.$, vol. 9, p. 808). Récemment, le gène de susceptibilité à la neurofibromatose du type 2 a été identifié comme une protéine du cytosquelette $(\mathrm{m} / \mathrm{s}$, $n^{\circ} 6 / 7$, vol. 9, p. 808). Enfin, les protéines des jonctions adherens sont la cible de tyrosine kinases, cytoplasmiques (c-Src) ou membranaires (récepteur de l'EGF). Cependant, des anomalies d'adhérence pourraient intervenir plus aux stades tardifs de la progression tumorale qu'à ses stades les plus précoces, comme cela est observé pour le gène $A P C$. Par conséquent, la prédisposition au cancer associée aux mutations du gène $A P C$ pourrait être due à une modification de la transmission d'un signal plutôt qu'à des anomalies d'adhérence des cellules.

A.K.

1. Leach FS, Nicolaides NC, Papadopoulos N, Liu B, Jen J, Parsons R, Peltomāki P, Sistonen P, Aaltonen LA, Nystróm-Lahti M. Mutations of a muts homolog in hereditary nonpolyposis colorectal cancer. (iell 1993; 75: $1215-25$.

2. Fishel R, Lescoe MK, Rao MRS, Copeland NG, Jenkins NA, Garber J, Kane M, Kolodner $\mathrm{R}$. The human mutator gene homolog MSH/2 and its association with hereditary nonpolyposis colon cancer. Ciell 1993; 75: $1027-38$.

3. Parsons R, Li GM, Longley MJ, Fang WH, Papadopoulos N, Jen J, de la Chapelle A, Kinzler KW, Vogelstein B, Modrich P. Hypermutability and mismatch repair deficiency in RER $^{+}$tumor cells. Cell 1993; 75: 1227-36. 4. Su LK, Vogelstein B, Kinzler KW. Association of the APC: tumor suppressor protein with catenins. Science 1993; 262: 1734-7.

5. Rubinfeld B, Souza B, Albert I, Müller (O, (hamberlain SH, Masiarz. FR, Munemitsu S, Polakis P. Association of the $A P($; gene product with $\beta$-catenin. Science 1993; 262: 1731-4.

6. Mège RM. Les molécules d'adhérence cellulaire: molécules morphogénétiques. médecine/sciences 1991; 7 : 544-52.

7. Peifer M. Cancer, catenins and cuticle pattern: a complex comnection. Science 1993; 262 : 1667-8. 